\title{
A Pneumatic Haptic Probe Replica for Tele-Robotized Ultrasonography
}

\author{
Ibrahim Abdallah ${ }^{1}$, Fabrice Gatwaza ${ }^{1}$, Nicolas Morette ${ }^{2}$, Arnaud Lelevé ${ }^{1}$, \\ Cyril Novales ${ }^{2}$, Laurence Nouaille ${ }^{2}$, Xavier Brun ${ }^{1}$, and Pierre Vieyres ${ }^{2}$ \\ 1 Univ Lyon, INSA Lyon, Laboratoire Ampère (UMR 5005), F - 69621, LYON, \\ France, \\ arnaud.leleve@insa-lyon.fr, \\ WWW home page: http://www.ampere-lab.fr \\ 2 Univ. Orléans, INSA-CVL, PRISME EA 4229, Bourges, France, \\ pierre.vieyres@univ-orleans.fr, \\ WWW home page: https://Www.univ-orleans.fr/prisme
}

\begin{abstract}
This paper introduces a pneumatic haptic device to remotely control a slave ultrasound probe-holder robot. This device should orientate this probe according to the sonographer's examination needs, while rendering the force applied by it on the patient's body, in order to provide a realistic examination environment as in situ. Previous designs with electric actuators were limited in terms of torque, dimensions and ergonomics, which actually did not match end-users' remote ultrasonography requirements. This paper describes the mechatronic design of an haptic pneumatic probe replica and preliminary control laws for it to perform as a Variable Stiffness Actuator (VSA). This approach is original and experimental results are provided to validate its feasibility.
\end{abstract}

Keywords: Medical Robotics, Haptics, Variable Stiffness Actuator, Pneumatics

\section{Introduction}

Nowadays, more than one out of four emergency admissions requires an ultrasound examination. This non-radiative and relatively low-cost imaging technique is routinely used to help physicians to deliver a preliminary diagnosis. Depending on state health policies, an ultrasound imaging diagnosis is performed either by trained physicians or by specialized sonographers. In both cases, the physician/sonographer must be close to the patient to maintain and hold the ultrasound probe on the designated anatomic area to perform the examination. The sonographer integrates the position of the probe and the motion of his hand to analyze the resulting 2D ultrasound images. Since the late 1990s, in order to deliver equitable healthcare in medically isolated settings, several concepts of remote robotized ultrasonography have been developed, giving the sonographer the ability to move an ultrasound probe on a distant patient $[7,20,10,5]$. TER [22] or Masuda [11] used fixed robots attached to a table. Current trends are light 
body-mounted robots [13,14]: a paramedic holds the robot on the patient body while the distant sonographer controls the probe orientation using a dedicated input device, as in Figure 1.

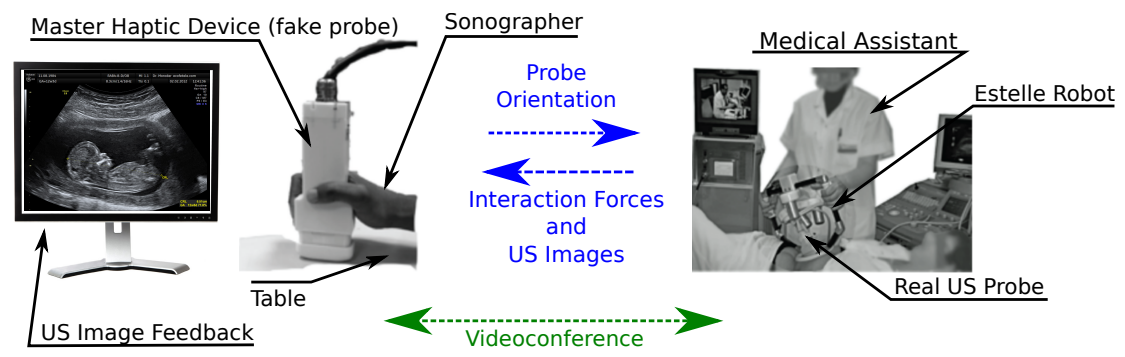

Fig. 1. Remote Echography.

Ergonomics is a critical requirement as the sonographers should not be disturbed by the distance with their patient in order to only focus on the medical procedure. Hence, master devices have to provide sonographers with full transparency to perform a robotized remote ultrasound scanning as if they were next to the patient. The master device must be adapted to the sonographer's hand and to his/her expertise. Devices such as SensAble Phantom perform 3D/6D motions and can render force feedback [5]. However, their kinematic chain is totally different from the one offered by the standalone ultrasound probes sonographers are used to. This means that the practitioner has to adapt his/her hand motions to the proposed input devices, which therefore disturbs the medical act. We proposed in [6] to provide the sonographer with a master ultrasound probe with no mechanical link with the environment, similar to a standard ultrasound probe. The practitioner was able to hold it and move it (on a table) as he/she would manipulate a real ultrasound probe on a patient body. With this master probe concept, trained doctors or specialized sonographers should need less training to control the distant robot [19].

Also, when performing a robotized ultrasound examination, sonographers need to feel the interaction between the ultrasound probe and the patient's body. Indeed, they need to feel when they touch hard body parts and when the body-probe interaction stiffness changes. This is even more true at distance as the practitioner does not have a direct view of the patient (only through a web camera). The master probe should thus be actuated to render the interaction forces (and stiffness) between the real remote ultrasound probe and the patient's body. This is a real ergonomic and technological challenge: it is important to preserve weight and dimensions comparable to standard ultrasound probes. We identified the following requirements:

- a reversible mechanism with small dimensions $(12 \mathrm{~cm}$ long, $6.5 \mathrm{~cm}$ wide and $3.5 \mathrm{~cm}$ thick at most), 
- in the $z$ direction (orthogonal to the patient's skin), a continuous force feedback level around $15 \mathrm{~N}$,

- a maximum force of $25 \mathrm{~N}$, a stroke of $50 \mathrm{~mm}$, with a maximum velocity of $200 \mathrm{~mm} / \mathrm{s}$.

The devices we could find in the literature use various small electric actuators with the lowest possible inertia, but are unsuccessful in meeting all the aforementioned requirements. Neither a DC-motor [12], nor a custom brushless motor [4], nor a linear motor [21] were able to provide the combined characteristics. For instance, when using standard electric actuators, planetary or harmonic gears have a reduction factor of $n$ for the rotation velocity which magnifies the inertia of the rotor by a factor $n^{2}$; this leads to the equivalent inertia of a $20 \mathrm{~kg}$ mass which makes the probe too sluggish.

In this paper, we propose to use a pneumatic cylinder as actuator to satisfy the end-users' requirements for the haptic input device to perform remote robotized ultrasonography. This approach is novel for this kind of application. This device is designed to be introduced in a bilateral control scheme (proposed earlier in [20]), controlling a remote robot which holds a real ultrasound probe. Performances of some basic control laws are evaluated in order to render, at the sonographer (master) site, the force applied on the remote patient's body by the real ultrasound probe, and the stiffness rendering.

The paper is organized as follows: section 2 introduces the tele-echography system used in this project. Section 3 introduces the new mechanical design and section 4 provides experimental results.

\section{The Melody Teleoperation System}

The tele-echography device developed by PRISME laboratory (see Figure 2) was industrialized under patent in a French company called AdEchoTech. The Melody robot dedicated to tele-echography is now commercialized for hospitals. The system is divided into two parts: a slave robot on the patient side and a handfree probe replica on the sonographer side without any mechanical connection between the two. A TCP/IP connection links the two parts.

In order to perform a haptic control, we enhanced the aforementioned Melody robot by replacing the electric motor which performs the longitudinal $(z)$ real probe motion by a linear motor. This ensures the reversibility of this motion and provides enough power (velocity and contact force) without any gear reducer. Moreover we added a force sensor which measures the reactive force of the patient's body on the ultrasound probe.

On the sonographer's side, we first designed electrically actuated probe replicas. Unfortunately, the various designs (with DC [12], brushless [21], custom direct drive or linear [21] motors) were mechanically irreversible, provided too slow dynamics, or were too heavy. Indeed, electrical motors struggle to provide linear fast motions on the one hand, and high forces at low speed on the other hand, all in a light and compact design without heating issues. This is why we propose a pneumatic actuation. 

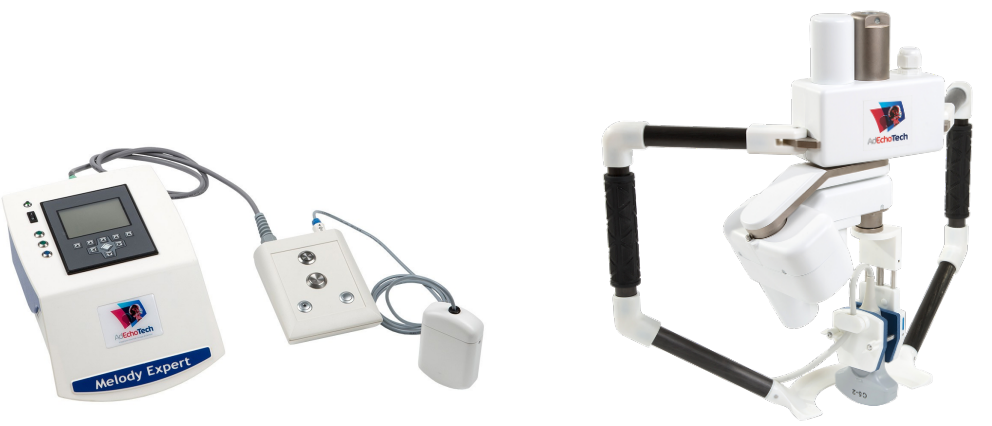

Fig. 2. Adechotech Melody system.

\section{Design of a Pneumatic Haptic Probe Replica}

\subsection{Variable Stiffness Actuation}

In order to reproduce a variable stiffness with an actuator, many Variable Stiffness Actuator (VSA) solutions have been proposed in the literature $[3,8,9]$. However, these solutions do not fit the sonographers' and remote ultrasound scanning requirements. Unlike electric actuators, pneumatic cylinders are lowcost off-the-shelf components and are easy to embed. They provide a natural passive compliance due to the pressurized air contained inside their two chambers. Moreover, by modifying these pressure levels, one can vary the pneumatic stiffness in real-time over a large range of values starting from $0.1 \mathrm{~N} / \mathrm{mm}$ according to [18].

For instance, Semini et al. [16] introduced a VSA based on position-controlled hydraulic cylinders. Abry et al. [1] proposed a finer control law to handle the global closed loop compliance of a pneumatic cylinder using a backstepping approach. In this case, high frequency disturbances are absorbed by the natural compliance of the pressurized chambers while the control loop adjusts its stiffness. Senac et al. [17] reused these works to design a syringe simulator in the context of epidural needle insertion simulation. This approach will be adapted later to the needs of our project to enhance its performance.

\subsection{Pneumatic Design}

Pneumatic cylinders offer non-linear dynamics, which require more complex control laws, but on the other hand, provide a natural compliance due to the compressibility of the air in the chambers. This compliance is an important property that we expect from this haptic interface in order to satisfy the force rendering requirements. Position or force control of pneumatic cylinders is well mastered nowadays in the fluid power community, as detailed in $[2,15]$.

In order to provide a guided active translation in the longitudinal direction between the socket and the body of the master probe, one asymmetric pneumatic 
cylinder (Pneumatic Union@) CS 10 E) was integrated beside a low friction slider. The cylinder rod is linked with the socket (see Figure 3). Two miniature piezoelectric 100 PSI pressure sensors (40PC series from Honeywell@), Canada) are located near the cylinder supplies through a $\mathrm{T}$ air connection to measure the cylinder chamber pressures. This is the closest location we could find with off-theshelf pneumatic components. The rod position is provided by a lightweight $(20 \mathrm{~g})$ position sensor (LP804-2 by Omega Engineering) located beside the cylinder. Its range is $51 \mathrm{~mm}$. The cylinder is supplied with a 5:2 Festoß MPYE-5-M5-010-B servovalve. A dSPACER DS1104 board is used to control the servovalve and to sample the sensor values.

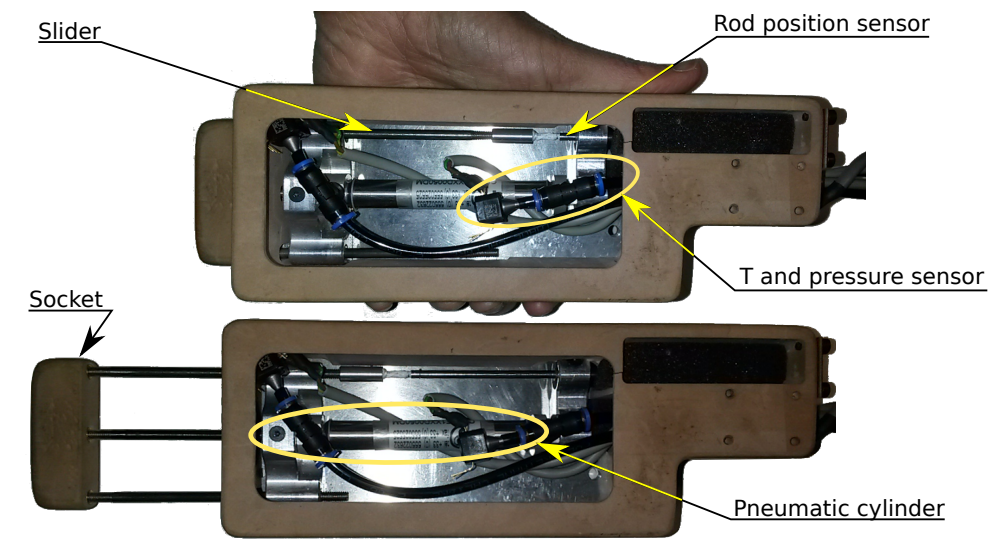

Fig. 3. Haptic pneumatic probe (with the rod completely in (top picture) and out (bottom picture).

The whole probe measures $120 \mathrm{~mm}$ high, $65 \mathrm{~mm}$ wide and $35 \mathrm{~mm}$ deep, and weighs $240 \mathrm{~g}$. It is bulky compared to modern ultrasonography probes. Nevertheless, this prototype was designed for the purposes of a feasibility study. Hence off-the-shelf low-priced components have been preferred over ergonomic constraints. Future designs will take ergonomics into account more comprehensively.

\subsection{Haptic Interface Force Control}

In order to feed back interaction forces from the slave robot, the pneumatic actuator has to be controlled accordingly. A low level force tracking control has been set up. It controls the force exerted by the pneumatic actuator on the probe socket. As the socket of the probe is held in contact with a stable horizontal surface, the reaction force makes the upper part of the probe shift it up, in order to reproduce the variable interaction force between the real patient and the real ultrasound probe measured on the remote robot. 
The global vertical (positive upward) force exerted on the hand of the practitioner is given by:

$$
f_{g}(t)=f_{p}(t)-b z_{m}(t)-f_{d r y}(t)-M_{u} g
$$

where $f_{p}$ is the force exerted by the pneumatic cylinder on the rod (in the extension direction of the rod), $b$ the viscous friction coefficient, $z_{m}$ the position of the upper part $\left(z_{m}=0\right.$ when the rod is completely retracted, $m$ subscript means "master"), $f_{d r y}$ the dry friction force, $M_{u}$ the mass of the upper part of the probe and $g$ the acceleration of gravity. Note that the $M_{u} g$ component is null when the rod is completely retracted as the upper part rests on the bottom part and the upper and lower parts rest on the force sensor.

The controller must track a desired force $f_{d}(t)$. To feed back this force (and reciprocally, the force exerted by the probe replica on the hand of the holder), the probe must push on a force sensor, attached to the horizontal surface, which measures the force applied by the probe on the table. The measured force also includes the weight of the replica but the main internal disturbances including frictions are thus naturally rejected in the control loop.

\section{Experimental validation}

\subsection{Force Tracking}

Experiments were performed with a PI controller $\left(K_{p}=0.1\right.$ and $K_{i}=4.10^{-3}$, see Figure 4). The probe replica was held by an operator, standing on a force sensor (a \pm 100 N ELPF Load Cell model, manufactured by Measurement Specialties, Inc.) as visible in Figure 5. So the force measured by the sensor $f_{m}$ is directly fed back to the controller. Note that in the following measures, the mass of the replica was not accounted for but it will be so in the future.

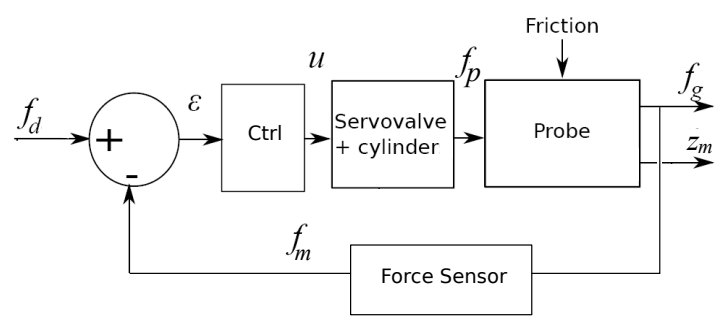

Fig. 4. Force controller for the master probe.

To evaluate the force tracking in realistic conditions (see next section), the desired force $f_{d}$ was expressed as a function of the vertical position of the probe as:

$$
f_{d}(t)=K_{d}\left(z_{m_{\text {out }}}-z_{m}(t)\right)
$$


where $K_{d}=400 \mathrm{~N} / \mathrm{m}$ was the desired stiffness, $z_{m_{\text {out }}}=50 \mathrm{~mm}$ is the position of the probe when the rod is completely out and $z_{m}$ is its instantaneous position measured by the probe internal position sensor. The supply pressure was 4 bar.

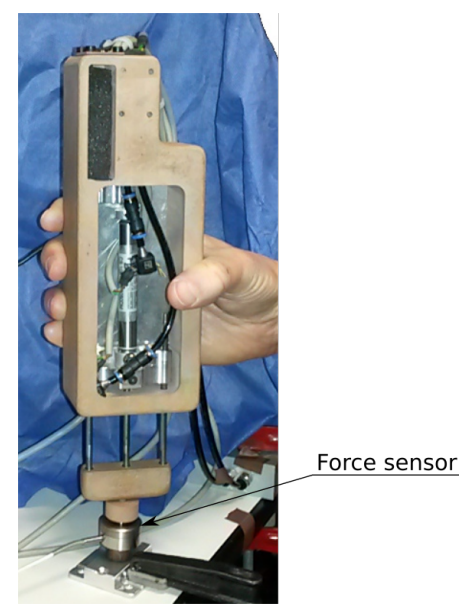

Fig. 5. Experimental Setup: the probe held by an operator and standing on a force sensor.

Figure 6 displays the desired and measured interaction forces, the tracking error and the position of the probe. One can observe a globally correct force tracking obtained with a simple PI controller. The worst performances are obtained for small force values below $10 \mathrm{~N}$ where the relative error rises up to $50 \%$. Over $10 \mathrm{~N}$, the mean relative error is around $10 \%$.

The probe can render larger forces: during the experiments, a force of $27 \mathrm{~N}$ could be obtained. The maximal speed was not evaluated during these experiments. The performances in terms of force tracking are mitigated. Higher gains generate oscillations. A better tracking control should be tested with nonlinear controllers such as in [17]. Also, the noise visible in the force error signal of Figure 6 results from a small sampling resolution of the force sensor signal (ADC range is $\pm 10 \mathrm{~V}$ while the sensor signal uses a range of $[0 \mathrm{~V}, 1.6 \mathrm{~V}]$ for this application). An analogic preamplification of this signal should diminish it.

\subsection{Stiffness Reproduction}

In addition to feedback interaction forces, the master probe, handled by the sonographer, must reproduce a desired stiffness estimated by the slave robot, equipped with a force sensor measuring the interaction forces between the skin and the real ultrasound probe. The desired stiffness estimated by the slave robot site is as follows: 

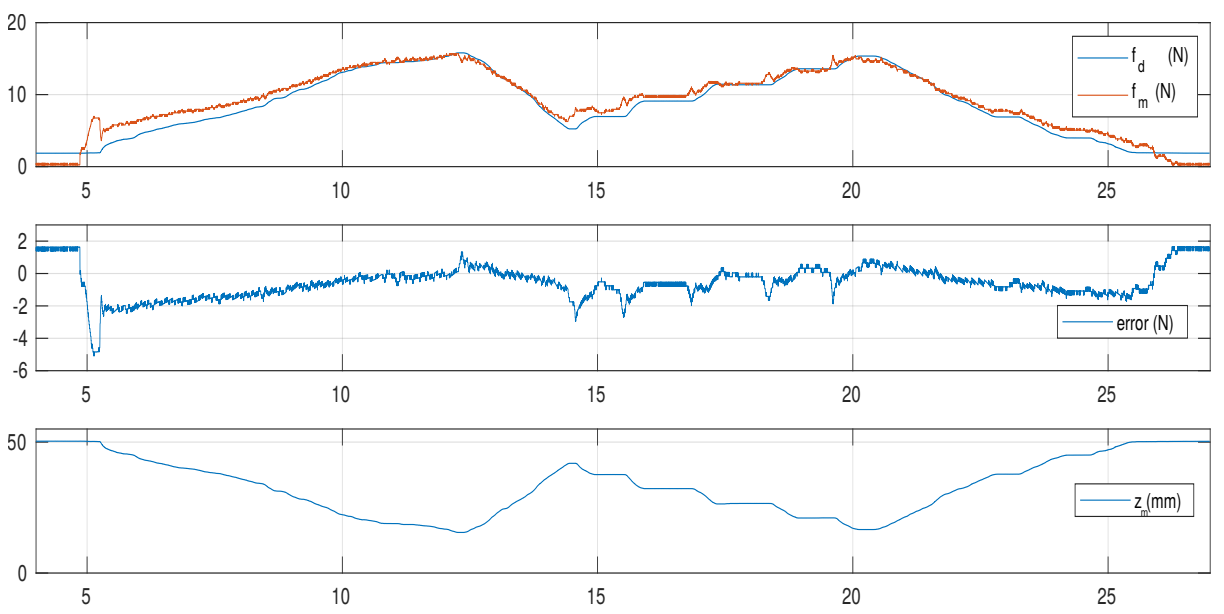

Fig. 6. Force Tracking Experimental Results.

$$
K_{d}=\frac{\Delta f_{e}}{\Delta z_{s}}
$$

where $\Delta f_{e}$ is the variation of the measured force applied by the slave robot on the patient skin, and $\Delta z_{s}$ is the variation of the position of the real probe in the axis orthogonal to the patient skin ( $z$ grows when the probe pushes harder against the skin), between each sample. This raw estimation should be further filtered and frozen when there is no motion. The estimated stiffness is transmitted through a TCP/IP network (satellite private link, Internet, etc). The controller of the master probe then computes a desired force to be applied by the actuator $f_{d}$ following eq. (2).

To evaluate the performance of the probe, we successively applied a range of desired stiffness $(50,100,200,400,800,1500 \mathrm{~N} / \mathrm{m})$ and asked the operator to "palpate" the table through the force sensor (see Figure 7). We reconstructed the apparent stiffness by rearranging eq. (2) :

$$
K_{\text {eval }}=-\frac{\Delta f_{m}}{\Delta z_{m}}
$$

where $\Delta f_{m}$ is the variation of the force measured by the force sensor on the master side, and $\Delta z_{m}$ is the variation of the position of the probe replica in the axis orthogonal to the patient skin, between each sample. After having removed some outliers due to short times where the probe did not move, the relative mean error $R M E$ is less than $4 \%$ (see eq (5) and Table 1). Taking into account that this stiffness reproduction is performed by force tracking, not by stiffness tracking, the resulting performance is satisfactory.

$$
R M E=\frac{\overline{\left|K_{\text {eval }}-K_{\text {des }}\right|}}{K_{\text {des }}}
$$




\begin{tabular}{|c|c|}
\hline$K_{\text {des }}[\mathrm{N} / \mathrm{m}]$ & $R M E(\%)$ \\
\hline 50 & 1.3 \\
100 & 1.7 \\
200 & 1.8 \\
400 & 1.8 \\
800 & 2.0 \\
1500 & 3.3 \\
\hline
\end{tabular}

Table 1. Relative Mean error for various values of desired stiffness.

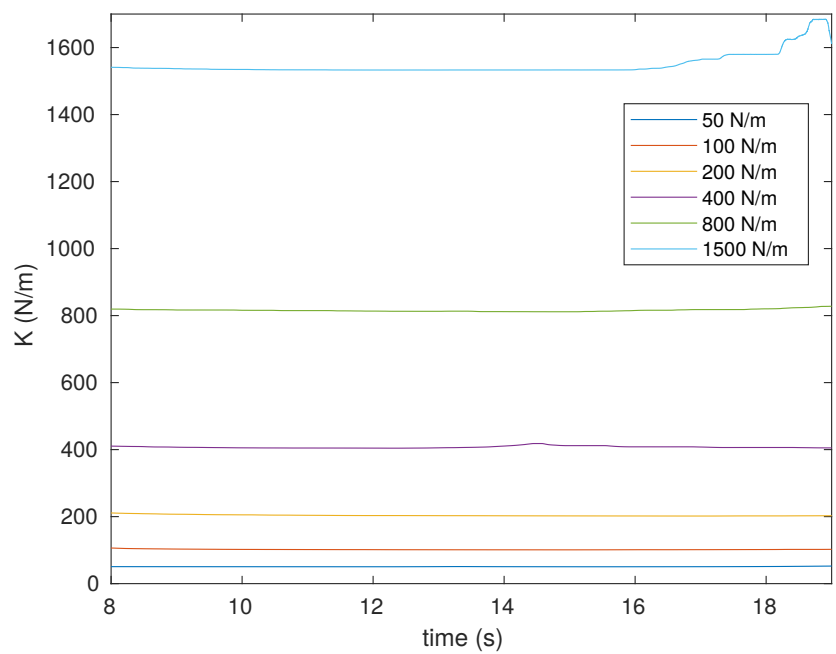

Fig. 7. Resulting stiffness for $K_{\text {des }} \in 50,100,200,400,800,1500 \mathrm{~N} / \mathrm{m}$.

\section{Conclusion}

This paper introduced a prototype of a pneumatic haptic probe to be used as a master input device in a teleoperated robotized ultrasonography system. Previous attempts to build hands-free haptic probes with electric actuators did not meet the end-users' specifications, which led us to study a pneumatic actuation. As the experimental results show, the use of a pneumatic actuator controlled as a Variable Stiffness Actuator makes it possible to reproduce a desired stiffness. Still, the force tracking is not yet sufficiently precise. This performance may still be enhanced by choosing a robust nonlinear controller such as in [17]. The reversibility of the probe replica, its strength $>25 \mathrm{~N}$, its stroke of $50 \mathrm{~mm}$ and its maximum velocity $>200 \mathrm{~mm} / \mathrm{s}$ already comply with our mechanical requirements. It is still a little over-sized, as off-the-shelf devices were used. However, 
this should be solved by selecting some smaller parts and/or custom-building other ones. Also, the use of this solution will require use of a compressed air supply. When not available, a compressed air cylinder can be used for this purpose. A study of the air consumption during typical ultrasonography sessions performed by radiologists will help us to determine its required capacity. Future experimentations featuring the whole teleoperation loop will be held to evaluate the overall quality of the whole system. Also, these results will be accompanied by a psychometric study determining whether users are able to recognize common medical cases.

\section{References}

1. Frederic Abry, Xavier Brun, Sylvie Sesmat, and Eric Bideaux. Non-linear position control of a pneumatic actuator with closed-loop stiffness and damping tuning. In Proc. of the European Control Conference 2013, 2013.

2. J.Falcao Carneiro and F. Gomes de Almeida. Using two servovalves to improve pneumatic force control in industrial cylinders. The International Journal of Advanced Manufacturing Technology, 66(1-4):283-301, 2013.

3. M. Cestari, D. Sanz-Merodio, J.C. Arevalo, and E. Garcia. Ares, a variable stiffness actuator with embedded force sensor for the atlas exoskeleton. Industrial Robot: An International Journal, 41(6):518-526, 2014.

4. G. Charron, N. Morette, T. Essomba, P. Vieyres, J. Canou, P. Fraisse, S. Zeghloul, A. Krupa, and Ph.Arbeille. Robotic platform for an interactive tele-echographic system: The prosit anr-2008 project. In Proc. of Hamlyn Symp. on Medical Robotics, London, UK, May 2010.

5. François Conti, Jaeheung Park, and Oussama Khatib. Proc. of the 12th International Symposium on Experimental Robotics, chapter Interface Design and Control Strategies for a Robot Assisted Ultrasonic Examination System, pages 97-113. Springer Berlin Heidelberg, Berlin, Heidelberg, 2014.

6. F. Courreges, C Novales, G. Poisson, and P. Vieyres. Modelisation, commande geometrique et utilisation d'un robot portable de tele-echographie: Teresa. Journal Europeen des Systemes Automatises (JESA), 43(1):165-196, March 2009. ISSN 12696935.

7. A. Gourdon, P. Poignet, G. Poisson, Pierre Vieyres, and P. Marche. A new robotic mechanism for medical application. In Proc. of the IEEE/ASME International Conference on Advanced Intelligent Mechatronics (AIM 1999), pages 33-38, 1999.

8. S.S. Groothuis, G. Rusticelli, A. Zucchelli, S. Stramigioli, and R. Carloni. The variable stiffness actuator vsaut-ii: Mechanical design, modeling, and identification. Mechatronics, IEEE/ASME Transactions on, 19(2):589-597, April 2014.

9. A. Jafari, N.G. Tsagarakis, I. Sardellitti, and D.G. Caldwell. A new actuator with adjustable stiffness based on a variable ratio lever mechanism. Mechatronics, IEEE/ASME Transactions on, 19(1):55-63, Feb 2014.

10. A. Krupa, D. Folio, C. Novales, P. Vieyres, and T. Li. Robotized tele-echography: An assisting visibility tool to support expert diagnostic. Systems Journal, IEEE, $\operatorname{PP}(99): 1-10,2014$.

11. K. Masuda, E. Kimura, N. Tateishi, and K. Ishihara. Three dimensional motion mechanism of ultrasound probe and its application for tele-echography system. In Proc. of the IEEE/RSJ International Conference on Intelligent Robots and Systems, volume 2, pages 1112-1116, 2001. 
12. Gilles Mourioux, Cyril Novales, Natalie Smith-Guerin, Pierre Vieyres, and Gerard Poisson. A free haptic device for tele-echography. In Proc. of International Workshop on Research and Education in Mechatronics (REM'2005), Annecy, June 2005.

13. Farshid Najafi and Nariman Sepehri. A novel hand-controller for remote ultrasound imaging. Mechatronics, 18(10):578 - 590, 2008.

14. L. Nouaille, P. Vieyres, and G. Poisson. Process of optimisation for a 4 dof teleechography robot. Robotica, 30:1131-1145, 122012.

15. Ramhuzaini Abd. Rahman, Liang He, and Nariman Sepehri. Design and experimental study of a dynamical adaptive backstepping?sliding mode control scheme for position tracking and regulating of a low-cost pneumatic cylinder. International Journal of Robust and Nonlinear Control, 26(4):853-875, 2016.

16. C. Semini, N.G. Tsagarakis, E. Guglielmino, M. Focchi, F. Cannella, and D.G. Caldwell. Design of hyq - a hydraulically and electrically actuated quadruped robot. Syst. Control Eng., 225:831-849, 2011. Proc. Inst. Mech. Eng. Part J.

17. Thibaut Senac, Arnaud Lelevé, and Richard Moreau. Control laws for pneumatic cylinder in order to emulate the loss of resistance principle. In IFAC 2017 World Congress, Proc. of the 20th World Congress of the International Federation of Automatic Control, Toulouse, France, July 2017. IFAC, IFAC.

18. M. Takaiwa and T. Noritsugu. Development of pneumatic human interface and its application for compliance display. In Proc. of 26th Annual Conference of the IEEE Industrial Electronics Society (IECON 2000), pages 806-811 vol.2, 2000.

19. P. Vieyres, C. Novales, R. Rivas, L. Vilcahuaman, J. Sandoval, T. Clark, K. DeStigter, L. Josserand, Z. Morrison, A. Robertson, S. Avgousti, N. Morette, S. Voskarides, A Fonte, T. Kasparis, and E. Lefebvre. The next challenge for world wide robotized tele-echography experiment (wortex 2012): From engineering success to healthcare delivery. In Proc. of TUMI II, Congreso Peruano de Ingeniera Biomedical Bioingeniera, Biotecnologica y Fisica Medica, Lima, Peru, May 2013.

20. Pierre Vieyres, Gerard Poisson, Fabien Courreges, Natalie Smith-Guerin, Cyril Novales, and Philippe Arbeille. A tele-operated robotic system for mobile teleechography: The otelo project. In S.H. Istepanian, Robert, Swamy Laxminarayan, and Constantinos S. Pattichis, editors, M-Health, Topics in Biomedical Engineering, pages 461-473. Springer US, 2006.

21. Pierre Vieyres, Juan Sandoval, Laurence Josserand, Cyril Novales, Marco Chiccoli, Nicolas Morette, Aicha Fonte, Soteris Avgousti, Sotos Voskarides, and Takis Kasparis. An anticipative control approach and interactive gui to enhance the rendering of the distal robot interaction with its environment during robotized tele-echography: Interactive platform for robotized tele-echography. Int. J. Monit. Surveill. Technol. Res., 1(3):1-19, 2013.

22. Adriana Vilchis Gonzales, Philippe Cinquin, Jocelyne Troccaz, Agnes Guerraz, Bernard Hennion, Franck Pellissier, Pierre Thorel, Fabien Courreges, Alain Gourdon, Gerard Poisson, Pierre Vieyres, Pierre Caron, Olivier Merigeaux, Loc Urbain, Cedric Daimo, Stephane Lavallee, Philippe Arbeille, Marc Althuser, JeanMarc Ayoubi, Bertrand Tondu, and Serge Ippolito. Ter: A system for robotic tele-echography. In WiroJ. Niessen and MaxA. Viergever, editors, Medical Image Computing and Computer-Assisted Intervention (MICCAI 2001), volume 2208 of Lecture Notes in Computer Science, pages 326-334. Springer Berlin Heidelberg, 2001. 